\title{
Abuso físico y sexual durante la niñez y revictimización de las mujeres mexicanas durante la edad adulta
}

\author{
Leonor Rivera-Rivera, M en C,(I) Betania Allen, M en Antrop,(I) \\ Rubén Chávez-Ayala, M en Psicol, ${ }^{(1)}$ Leticia Ávila-Burgos, Dr en Econ. ${ }^{(2)}$
}

\begin{abstract}
Rivera-Rivera L, Allen B, Chávez-Ayala R, Avila-Burgos L. Abuso físico y sexual durante la niñez y revictimización de las mujeres mexicanas durante la edad adulta. Salud Publica Mex 2006;48 supl 2:S268-S278.
\end{abstract}

\section{Resumen}

Objetivo. Cuantificar la asociación que existe entre el abuso físico y sexual durante la niñez y la violencia durante la edad adulta en una muestra representativa de usuarias de los servicios de salud en México. Material y métodos. Se analizaron datos provenientes de una encuesta nacional, con una muestra de 26042 mujeres mayores de 14 años, quienes acudieron a consulta a los servicios públicos de salud entre octubre de 2002 y marzo de 2003, en los 32 estados de la República. Se utilizaron dos modelos: a) Modelo de regresión logística politómica múltiple para explorar las asociaciones entre violencia física y sexual por parte de la pareja y violencia durante la niñez. b) Modelo de regresión logística múltiple para evaluar la asociación entre violación durante la edad adulta y violencia durante la niñez. Resultados. Se encontró una asociación entre experimentar violencia física durante la niñez, y padecer violencia física y sexual por parte de la pareja o sufrir violación durante la edad adulta. Cuando la mujer informó que había recibido golpes "casi siempre" durante la niñez, era más probable que experimentara violencia física y sexual $(R M=3$.I; IC95\% 2.6-3.7) y violación ( $R M=2.9 ;$ IC95\% 2.4-3.6) durante la edad adulta. Además, mientras más frecuente había sido la violencia durante la niñez, era mayor la posibilidad de que la mujer sufriera violencia posteriormente. Asimismo, se encontró una asociación positiva entre violencia física y sexual por parte de la pareja y abuso sexual antes de los 15 años de edad. ( $R M=2.8$; IC95\% 2.2-3.5). La violación sufrida en la edad adulta también estuvo asociada al abuso sexual antes de los 15 años de edad (RM=II.8; IC95\% I0.2-I3.7). Con-
Rivera-Rivera L, Allen B, Chávez-Ayala R, Avila-Burgos L. Physical and sexual abuse during childhood and revictimization during adulthood in Mexican women. Salud Publica Mex 2006;48 suppl 2:S268-S278.

\section{Abstract}

Objective. To quantify the association between physical and sexual abuse during childhood and violence during adulthood in a representative sample of female health care users in Mexico. Material and Methods. A questionnaire was administered to 26042 women over 14 years of age who sought medical consultation from public health care services between October 2002 and March 2003, in all 32 states in Mexico. Two models were constructed: a) Multiple polytomic logistic regression models to explore the association between violent victimization by the partner during adulthood and violence during childhood. b) Multiple logistic regression models to explore the association between experiencing rape during adulthood and violence during childhood. Results. Among women studied, an association was found between experiencing physical violence during childhood and suffering physical and sexual violence from the male partner or experiencing rape, during adulthood. When physical violence during childhood occurred "almost always", it was more likely that the woman undergo physical and sexual violence $(O R=3.1 ; 95 \% \mathrm{Cl} 2.6-3.7)$ and rape $(O R=2.9 ; 95 \% \mathrm{Cl} 2.4-3.6)$, during her adult life. In addition, when violence during childhood was more frequent, the likelihood of experiencing violence during adulthood was greater. A positive association was found between physical and sexual abuse before 15 years of age $(O R=2.8 ; 95 \% \mathrm{Cl} 2.2-3.5)$. Experiencing rape during adulthood was also associated with sexual abuse before 15 years of age $(O R=|| .8 ; 95 \% \mathrm{Cl}$ 10.2-13.7). Conclusions. In this sample of Mexican women, both physical and sexual violence during childhood has

(I) Dirección de Salud Reproductiva, Centro de Investigación en Salud Poblacional, Instituto Nacional de Salud Pública, Cuernavaca, Morelos, México.

(2) Dirección de Economía y Políticas en Salud, Centro de Investigación en Sistemas de Salud, Instituto Nacional de Salud Pública, Cuernavaca, Morelos, México.

Fecha de recibido: 26 de agosto de 2004 - Fecha de aprobado: 3I de mayo de 2005 Solicitud de sobretiros: Leonor Rivera-Rivera, Instituto Nacional de Salud Pública, Av. Universidad 655, col. Santa María Ahuacatitlán, 62508 Cuernavaca, Morelos, México. Correo electrónico: Irivera@correo.insp.mx 
clusiones. La violencia sufrida durante la niñez crea un cuadro de resultados negativos, tanto psicológicos como físicos, y uno de ellos es la revictimización. Es decir, las mujeres que son víctimas de violencia durante la niñez tienen una mayor probabilidad de sufrirla durante la edad adulta. El abuso físico y sexual durante la niñez debe prevenirse o, en su defecto, detectarse y tratarse.

Palabras clave: violencia familiar; violencia sexual; violencia de pareja; revictimización; México negative results during adulthood, including a greater likelihood of revictimization by the male partner and rape. Physical and sexual abuse during childhood must be prevented or at least detected and treated.

Key words: family violence; sexual violence; partner violence; revictimization; Mexico
A lo largo de su vida, las mujeres pueden verse afectadas por la violencia. Durante la infancia pueden sufrir violencia en el hogar por parte de familiares. En etapas posteriores, existe la posibilidad de que sufran violencia, ya sea por parte de familiares y conocidos en sus hogares, como por desconocidos en el ámbito público. Los perpetradores suelen ser miembros de la familia -especialmente padres-, la pareja masculina y, en ocasiones, personas desconocidas (estas últimas cuando se trata principalmente de abuso sexual, aunque esto no significa que no exista violencia sexual por parte de personas cercanas).

Diversos estudios indican que la experiencia de abuso físico o sexual sufrido durante la niñez provoca resultados negativos en diversos ámbitos de la vida adulta. Algunos de estos resultados tienen consecuencias en la salud física, como, por ejemplo, presentar más problemas de salud en general, ${ }_{1}^{1}$ padecer fatiga crónica ${ }^{2}$ o enfermedades gastrointestinales. ${ }^{3}$ Otras consecuencias de la violencia sufrida durante la niñez son psicológicas, como baja autoestima y depresión, ${ }^{4-6}$ así como la ideación suicida y los intentos de suicidio. ${ }^{7-10}$ Asimismo, diversas adicciones como el tabaquismo, el abuso en el consumo de alcohol y el uso de drogas ilegales $^{11,12}$ son más frecuentes entre las personas que sufrieron abuso durante la niñez. Experimentar abuso físico o sexual durante la infancia también se asocia con embarazos no deseados, tener múltiples parejas sexuales sin protección y otros comportamientos que implican riesgos relacionados con infecciones de transmisión sexual, incluyendo el VIH/SIDA. ${ }^{13-15}$

Además, múltiples estudios muestran que las mujeres que son víctimas de violencia física en etapas tempranas de su vida (usualmente perpetrada por familiares) tienen mayor probabilidad de ser víctimas de este tipo de violencia en etapas posteriores de su vida, en las que el principal agresor suele ser la pareja masculina. ${ }^{16-31}$ Algunas investigaciones han encontrado que las niñas que son víctimas de abuso sexual tienen ma- yor probabilidad de ser revictimizadas sexualmente en la vida adulta. ${ }^{16,32-35}$ Mientras que la victimización se concibe como el acto de recibir violencia (física, sexual o emocional), la revictimización se define como el hecho de que un individuo sea víctima de violencia interpersonal en dos o más momentos de la vida; ambas experiencias separadas por el tiempo y realizadas por parte de al menos dos perpetradores diferentes. Es decir, se refiere a sufrir abuso físico o sexual por parte de un familiar durante la niñez y luego experimentarlo nuevamente durante la vida adulta, cuando el perpetrador es la pareja masculina. ${ }^{36-38}$

Algunos estudios realizados con mujeres mexicanas han mostrado una asociación positiva entre violencia durante la niñez y violencia en la vida adulta. ${ }^{39-41}$ Sin embargo, son escasas las investigaciones en las que se haya estudiado el abuso sexual durante la niñez y su asociación con la violencia en la edad adulta. Por lo tanto, el objetivo del presente estudio ha sido, por una parte, cuantificar la asociación entre la experiencia del abuso físico y sexual durante la niñez, y, por la otra, el ser víctima de violencia (tanto física como sexual) en la vida adulta. Para que los hallazgos tuvieran una mayor representatividad, se decidió hacer el análisis de los datos proporcionados por una encuesta nacional, realizada con una muestra representativa de usuarias de los servicios de salud en la República mexicana.

\section{Material y métodos}

El presente estudio es parte de la Encuesta Nacional sobre Violencia contra las Mujeres 2003 (ENVIM 2003). En total, participaron 26042 mujeres de 15 y más años de edad, las cuales fueron seleccionadas de manera aleatoria de entre las usuarias de instituciones de salud pública que utilizaron servicios de primer y segundo niveles de atención, entre octubre de 2002 y marzo de 2003, en las 32 entidades federativas de México. El tipo de muestreo fue probabilístico y estratificado en dos 
etapas. En la primera se seleccionaron unidades médicas con probabilidad proporcional al número de consultorios de la unidad, de entre un listado de las unidades seleccionadas. En la segunda etapa se seleccionaron mujeres mayores de 14 años de edad que acudieron a consulta médica. La selección de las mujeres se realizó utilizando un muestro sistemático.

Para llevar a cabo el levantamiento de las encuestas fue necesario capacitar a un grupo de entrevistadoras durante tres meses. La capacitación del personal se llevó a cabo en dos etapas. La primera consistió en el manejo del cuestionario y de las técnicas de entrevista. En la segunda etapa se capacitó a las entrevistadoras en técnicas para el manejo de mujeres en situación de violencia y revisión de las definiciones de los diferentes tipos de violencia. Se llevaron a cabo talleres de integración grupal y otras dinámicas de grupo.

En total, participaron 100 entrevistadoras entre enfermeras, trabajadoras sociales, psicólogas y sociólogas, además de 16 supervisores y 10 coordinadoras.

Las mujeres entrevistadas fueron captadas en los servicios de primer y segundo niveles de atención en urgencias, medicina interna y ginecología. Las entrevistas fueron realizadas en un espacio cerrado. Antes de iniciarlas, se entregó a las mujeres una carta de consentimiento informado, lo que garantizó la confidencialidad de la información.

Una vez terminadas las entrevistas, se entregó a las participantes un tríptico con información sobre violencia y un directorio de las instituciones que atienden a las mujeres en situación de violencia. Para mayor información sobre la metodología véase la ENVIM $2003 .{ }^{42}$

\section{Instrumento de recolección de información}

El cuestionario de la ENVIM 2003 estuvo integrado por 17 secciones; sin embargo, para fines del presente estudio, sólo se consideraron las siguientes secciones: identificación de los hogares, datos generales (edad, escolaridad, estado civil), características de la vivienda y hogar, experiencia de violencia por parte del esposo (actos de violencia ocurridos durante los últimos 12 meses) y otras experiencias de violencia (violencia durante la niñez). ${ }^{42}$

\section{Medición de las variables de estudio}

Violencia de pareja

De la conceptualización de la violencia como patrón de maltrato por parte de la pareja masculina hacia la mujer, y caracterizado por una serie de conductas dominan- tes y represivas, se construyó una escala de violencia, la cual consta de 27 reactivos que fueron seleccionados del Index of Spouse Abuse (ISA) y del Severity of Violence Against Women Scale (SVAWS) (anexo 1). ${ }^{43,44}$ Se preguntó sobre la frecuencia de actos de violencia que había sufrido la mujer durante los últimos 12 meses por parte de su pareja, compañero, esposo o novio. La escala de la ENVIM 2003 fue validada y se obtuvo un alfa de Cronbach de 0.99. ${ }^{45}$

La escala de violencia de pareja fue validada a través de dos procedimientos:

1. Análisis de las respuestas de mujeres de la ENVIM 2003.

2. Realización de un jueceo para asignar pesos diferenciales a cada uno de los reactivos de la escala, tomando en cuenta el daño físico y emocional de cada acción o comportamiento violento incluido en la escala.

Se solicitó a los jueces que consideraran, para cada una de las acciones evaluadas, dos dimensiones: el daño emocional y el daño físico. Se utilizó una escala de 1 a 10, en donde 1 es el nivel más bajo de daño y 10 el más alto. Cada una de las dimensiones fue evaluada por separado. Se calculó el promedio y la desviación estándar de los puntajes asignados por los jueces para cada reactivo.

Para el presente estudio, sólo se analizaron dos tipos de violencia por parte de la pareja: física y sexual.

a) Violencia física

Se consideró un total de 11 reactivos para la medición de violencia física: 1. ¿Le ha pegado con el puño o la mano? 2. ¿Le ha sacudido, zarandeado, o jaloneado? 3. ¿Le ha torcido el brazo? 4. ¿Le ha pateado? 5. ¿Le ha empujado a propósito? 6. ¿Le ha disparado con una pistola o rifle? 7. ¿Le ha agredido con navaja, cuchillo o machete? 8. ¿Le ha quemado con cigarro u otra sustancia? 9. ¿Le ha amenazado con pistola o rifle? 10. ¿Le ha amenazado con alguna navaja, cuchillo o machete? 11. ¿La ha tratado de ahorcar o asfixiar?

A cada reactivo se le asignó un puntaje de acuerdo con los resultados obtenidos por los jueces; posteriormente se sumaron todos los reactivos que corresponden a violencia física y se obtuvo un punto de corte a partir de la media. Los puntos de corte para considerar casos de violencia física fueron: ${ }^{45}$

- Por arriba de la media se consideró caso de violencia.

- Por debajo de la media se consideraron casos de no violencia. 
- Se obtuvo una variable dicotómica $0=$ no violencia física y $1=$ violencia física.

- Para obtener una variable dicotómica de violencia sexual, se realizó el mismo procedimiento que el utilizado para la violencia física.

b) Violencia sexual

Se consideraron tres reactivos para medir la violencia sexual por parte de la pareja: 1. ¿Le ha exigido tener relaciones sexuales? 2. ¿Ha usado la fuerza física para tener relaciones sexuales? 3. ¿Le ha amenazado con irse con otras mujeres si no accede a tener relaciones sexuales? ${ }^{45}$

Construcción de las variables dependientes

a) Violencia física y sexual por parte de la pareja

Una vez obtenidas las dos variables dicotómicas (violencia física y sexual), se realizaron combinaciones posibles, de tal forma que se obtuvo una variable en cuatro categorías: $0=$ no violencia, $1=$ violencia física y sexual, 2 = sólo violencia física, 3 = sólo violencia sexual.

b) Violación en la edad adulta

Se analizó también como variable dependiente la violación. Para medir la variable de violación, se preguntó a las usuarias de los servicios de salud lo siguiente: ¿Aparte de su esposo, compañero o última pareja, la han forzado a tener relaciones sexuales o a realizar algún acto sexual en contra de su voluntad? Esta variable se dicotomizó en $0=$ nunca y $1=$ si contestaron afirmativamente la pregunta (en esta opción de respuesta se incluye al padre, padrastro, otros miembros de la familia, profesor, policías, desconocidos, vecinos, sacerdotes).

Debido a que en la violación no se considera a la pareja como agresor, se realizó otro modelo para evaluar las variables asociadas a la misma.

\section{Variables independientes}

Violencia física durante la niñez

Se recolectó información sobre la violencia física que experimentó la mujer durante la niñez mediante la siguiente pregunta: Cuando era niña, ¿sus padres o familiares le pegaban? Se midió la frecuencia con la que ocurrió la violencia mediante la siguiente escala: $1=$ nunca, $2=$ pocas veces, $3=$ varias veces, $4=$ casi siempre.
Abuso sexual antes de los 15 años

Para conocer el antecedente de abuso sexual durante la niñez, se preguntó a las participantes si: Antes de los 15 años de edad, ¿usted recuerda si alguien la tocó alguna vez sexualmente o le hizo algo sexual en contra de su voluntad? Esta variable se clasificó en dos categorías: $0=$ nunca y $1=$ si contestaron afirmativamente la pregunta (en esta opción de respuesta se incluye a padres, padrastros, otros miembros de la familia y novio).

Nivel socioeconómico

La definición de los estratos socioeconómicos se basó en la combinación de tres características de los hogares: la escolaridad, la actividad de los miembros de la familia y los activos de la vivienda.

Se calculó el promedio para todos los miembros del hogar y se clasificó el nivel socioeconómico en cuatro categorías:

a) Muy bajo, cuando el valor obtenido en el promedio es de hasta -0.25 .

b) Bajo: valores de -0.26 hasta 0.1 .

c) Media: valores de 0.11 hasta 0.5 .

d) Alta: valores por arriba de 0.5 .

El desarrollo de este índice se explica en el artículo metodológico correspondiente. ${ }^{11}$

Otras variables de estudio

Se consideraron variables sociodemográficas como son: edad (en años cumplidos; para el análisis final se clasificó la edad en cuatro categorías: 15 a 25, 26 a 33, 34 a 44, y 45 o más); estado civil (casada o en unión libre, divorciada o separada, viuda o soltera); escolaridad (en años de estudio; posteriormente se clasificó la escolaridad en analfabetas, con primaria y secundaria, preparatoria o más).

\section{Análisis estadístico}

Se realizó un análisis univariado para ver la distribución de las variables de interés. Para identificar las variables asociadas con la violencia física y sexual por parte de la pareja, se realizó, primero, un análisis bivariado en el que se utilizó regresión logística politómica y se obtuvieron razones de momios $(R M)$ con intervalos de confianza de 95\% (IC95\%). Posteriormente, se realizó un análisis de regresión logística 
politómica múltiple para evaluar las posibles variables confusas. Los modelos finales estuvieron conformados por las variables que, en el análisis bivariado, presentaron un valor de $p<0.20$. Se realizó la prueba de Hosmer y Lemoshow para evaluar la bondad de ajuste del modelo final. El motivo de realizar análisis de regresión logística politómica fue que la variable dependiente de violencia de pareja fue conformada en cuatro categorías: $0=\sin$ violencia, $1=$ violencia física y sexual, $2=$ sólo violencia física y $3=$ sólo violencia sexual.

Para evaluar las variables asociadas con violación excluyendo a la pareja, se realizó otro análisis; sin embargo, debido a que nuestra variable de violación fue de tipo dicotómica, se realizó el análisis de regresión logística bivariado y, posteriormente, el de regresión logística múltiple para evaluar las posibles variables confusas.

El análisis se realizó con el paquete estadístico Stata versión 7.0.*

\section{Resultados}

\section{Características sociodemográficas}

La media de edad de las mujeres que participaron en el estudio fue de 35.8 años con una desviación estándar (DS) de 13.7. Al categorizar la edad, se observó que $26.8 \%$ de la población se encuentra en el grupo de 15 a 25 años de edad. En relación con el nivel socioeconómico, $45.3 \%$ de las participantes fueron de nivel muy bajo, $39.2 \%$ de nivel bajo y $15.5 \%$ de los niveles medio y alto. Con respecto a la escolaridad, la mayoría de las usuarias de los servicios de salud cursaron primaria y secundaria. El promedio de años de educación formal fue de 7.3 (DS=4.2).Un poco más de la mitad de las mujeres eran casadas $(56.0 \%)$, seguidas por las que vivían en unión libre $(18.0 \%)$, luego por las separadas, divorciadas o viudas $(14.2 \%)$ y finalmente las solteras $(11.7 \%)$ (cuadro I).

\section{Frecuencia de violencia en diferentes etapas de la vida}

A lo largo de su vida, las mujeres son víctimas de algún tipo de violencia. Por lo tanto, en el presente estudio se preguntó si las mujeres recibieron golpes por parte de los padres $u$ otros miembros de la familia. Casi la mitad contestó afirmativamente a esta pregunta $(42.5 \%)$. De este porcentaje, $24.5 \%$ de las mujeres contestaron que recibieron golpes pocas veces, $9.3 \%$ varias veces y $8.4 \%$ casi siempre (cuadro II).

\footnotetext{
* Stata 7.0 Software Stata Corp, College Station. TX.
}

Cuadro I
Características SOCIOdemográficas
De LA Población De estudio. ENVIM 2003, México
\begin{tabular}{lll} 
Variables & Número & Porcentaje \\
Edad en años & & \\
I5-25 & 6990 & 26.8 \\
\hline $26-33$ & 635 I & 24.4 \\
\hline $34-44$ & 6349 & 24.4 \\
\hline 45 o más & 6345 & 24.4
\end{tabular}

Nivel socioeconómico

\begin{tabular}{lrr} 
Muy bajo & 11753 & 45.3 \\
\hline Bajo & 10180 & 39.2 \\
\hline Medio y alto & 4031 & 15.5
\end{tabular}

Escolaridad

\begin{tabular}{lrr} 
Analfabeta & 2435 & 9.4 \\
\hline Primaria y secundaria & 17246 & 66.2 \\
\hline Preparatoria o más & 6361 & 24.4
\end{tabular}

Estado civil

\begin{tabular}{lrr} 
Casada & 14593 & 56.0 \\
\hline Unión libre & 4690 & 18.0 \\
\hline Separada, divorciada o viuda & 3704 & 14.2 \\
\hline Soltera & 3055 & 11.7
\end{tabular}

Acerca del abuso sexual antes de los 15 años de edad, en el presente estudio se encontró que 7.3\% de las mujeres respondieron afirmativamente a esta pregunta.

Como era de temerse, las mujeres continúan con el problema de violencia en la vida de pareja o adulta. Para fines de la presente investigación, se construyó una variable de violencia física y sexual, y se encontró que $4.9 \%$ presentó violencia física y sexual por parte de la pareja o esposo; $5.2 \%$ presentó sólo violencia física y 2.1\% sólo violencia sexual por parte de la pareja (cuadro II).

Asimismo, se observó que algunas mujeres han sufrido violación por personas distintas a la pareja; en el presente estudio, 3.4\% de las entrevistadas contestó afirmativamente a esta variable (cuadro II).

\section{Violencia durante la niñez asociada a violencia de la pareja actual}

Para evaluar el efecto que provoca la violencia durante la niñez en la violencia con la pareja actual, se realizó un análisis de regresión logística politómica múltiple, ajustado de acuerdo con la edad de las mujeres, nivel socioeconómico, escolaridad y estado civil.

salud pública de méxico / vol.48, suplemento 2 de 2006 


\section{Cuadro II \\ Frecuencia de violencia a lo largo \\ DE LA VIDA DE LAS MUJERES MEXICANAS. ENVIM 2003, MÉxICO}

\begin{tabular}{lrc} 
Tipo de violencia & Número & Porcentaje \\
$\begin{array}{l}\text { Golpes durante la niñez } \\
\text { Nunca }\end{array}$ & 15053 & 57.8 \\
\hline Pocas veces & 6382 & 24.5 \\
\hline Varias veces & 2412 & 9.3 \\
\hline Casi siempre & 2195 & 8.4 \\
$\begin{array}{l}\text { Abuso sexual (<15 años de edad) } \\
\text { No }\end{array}$ & & \\
\hline Sí & 24136 & 92.7 \\
\hline
\end{tabular}

\begin{tabular}{lrr}
$\begin{array}{l}\text { Violencia fisica y sexual por parte de la pareja } \\
\text { Sin violencia }\end{array}$ & 2 | 932 & 87.8 \\
\hline Violencia física y sexual & I 212 & 4.9 \\
\hline Sólo violencia física & I 295 & 5.2 \\
\hline Sólo violencia sexual & 534 & 2.1
\end{tabular}

Antecedente de violación*

\begin{tabular}{lrc} 
No & $25 \quad 165$ & 96.6 \\
\hline Sí & 877 & 3.4 \\
\hline Total & 26042 & 100
\end{tabular}

*Esta pregunta excluyó al esposo o la pareja

Se encontró una fuerte asociación entre experimentar golpes por parte de los familiares durante la niñez, y la violencia de pareja, aunque esta asociación se observa independientemente de la gravedad de la violencia física durante la niñez. Se observa la existencia de un claro gradiente de violencia de pareja; es decir, a medida que los golpes durante la niñez fueron más frecuentes, aumentó la posibilidad de sufrir violencia física y sexual por parte de la pareja actual. Así, la posibilidad de ser maltratada física y sexualmente por la pareja o esposo actual es de 1.44 veces más para las mujeres que refirieron que el maltrato por parte de familiares durante la infancia ocurrió pocas veces, y aumentó hasta 3.10 veces cuando este maltrato fue casi siempre (cuadro III).

Otra de las variables de violencia durante la niñez que mostró asociación positiva con violencia de pareja actual fue el abuso sexual antes de los 15 años de edad. Es decir, las mujeres que fueron víctimas de abuso sexual antes de los 15 años de edad tienen 3.10 veces más posibilidad de padecer violencia física y sexual con la pareja actual, en comparación con las mujeres que no sufrieron abuso sexual antes de los 15 años de edad. Es de llamar la atención qué importancia tiene el abuso sexual durante la niñez como predictor de violencia física y sexual en la vida adulta.

\section{Violencia durante la niñez asociada a violación en la vida adulta}

Los resultados del análisis de regresión logística múltiple ajustada por edad, nivel socioeconómico, escolaridad y estado civil, mostraron, como predictores de violación, la violencia intrafamiliar durante la niñez y el abuso sexual antes de los 15 años de edad. Podemos observar que, conforme los golpes son más frecuentes durante la niñez, aumenta la posibilidad de ser víctima de violación por una persona diferente al esposo o pareja (cuadro IV). Cuando los golpes durante la ni-

\section{Cuadro III \\ Efecto de la violencia dURANTE LA NIÑEZ en la violencia física y SeXual de la pareja actual. ENVIM 2003, México}

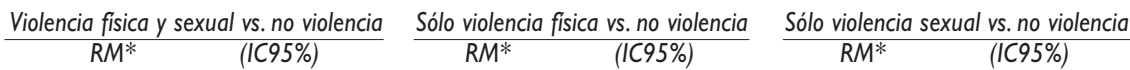

\begin{tabular}{|c|c|c|c|c|c|c|}
\hline \multicolumn{7}{|c|}{ Golpes durante la niñez } \\
\hline Nunca & 1.0 & & 1.0 & & 1.0 & \\
\hline Pocas veces & 1.44 & $1.23-1.67$ & 1.76 & $1.54-2.02$ & 1.82 & $1.48-2.23$ \\
\hline Varias veces & 3.13 & $2.07-2.96$ & 2.50 & $2.10-2.98$ & 1.87 & $1.42-2.48$ \\
\hline Casi siempre & 3.10 & $2.64-3.7 \mid$ & 2.41 & $2.00-2.90$ & 2.00 & $1.5 \mathrm{I}-2.65$ \\
\hline \multicolumn{7}{|c|}{ Abuso sexual $(<15$ años de edad) } \\
\hline No & 1.0 & & 1.0 & & 1.0 & \\
\hline Sí & 3.10 & $2.65-3.63$ & 1.98 & $1.67-2.36$ & 2.78 & $2.19-3.54$ \\
\hline
\end{tabular}


ñez ocurren pocas veces, la posibilidad de ser víctima de violación durante la vida adulta fue de 1.6 veces. En cambio, cuando los golpes durante la niñez ocurren casi siempre, la posibilidad de ser víctima de violación en la vida adulta es de 2.9 veces (cuadro IV).

Otra de las variables fuertemente asociadas con la violación en la vida adulta fue el abuso sexual antes de los 15 años. En el presente estudio se encontró que las mujeres que refirieron abuso sexual durante la niñez tuvieron 11.8 veces mayor posibilidad de padecer por lo menos una violación por parte de una persona diferente a la pareja, en comparación con las mujeres que no tuvieron este antecedente (cuadro IV).

\section{Discusión}

En el presente estudio se encontró una importante asociación entre el hecho de ser víctima de violencia física o abuso sexual durante la niñez y volver a experimentar la violencia física y sexual en la edad adulta. Como se comentó anteriormente, diversas investigaciones realizadas en otros países han encontrado también este fenómeno de repetición de la violencia en diferentes momentos de la vida y por perpetradores distintos, llamado revictimización. ${ }^{13,16-31,36,37}$ Este estudio es relevante porque indica que también en el contexto mexicano hay una asociación entre la violencia experimentada durante la niñez y la violencia sufrida en la vida adulta. Adicionalmente, la actual investigación muestra que existe una asociación específica entre el abuso sexual durante la infancia y la violencia (física y sexual) en la vida adulta, un tema que ha sido menos estudiado que el de la violencia física.
En el caso del abuso sexual durante la niñez y la revictimización con violencia física y sexual en la vida adulta, algunos autores han encontrado una asociación de este tipo, como encontramos en esta muestra de mujeres mexicanas. El presente estudio demuestra la existencia de una asociación significativa y evidente, ya que las mujeres que fueron abusadas sexualmente durante la niñez tuvieron 3.10 veces más posibilidad de ser víctimas de violencia física y sexual por la pareja, y 11.8 veces más posibilidad de sufrir violación por parte de una persona diferente a su pareja. De manera similar, Urquiza y colaboradores, ${ }^{33}$ que estudiaron una población estadounidense de mujeres de diversos grupos étnicos (blancas, negras, latinas y asiáticas), encontraron que las mujeres que habían sufrido abuso sexual durante la niñez tenían mayor probabilidad de ser violadas de adultas. En otro estudio realizado en jóvenes alemanas, se encontró que era más probable que las que notificaran abuso sexual durante la niñez también señalaran contacto sexual no deseado cuando eran adolescentes. ${ }^{34}$ Mientras tanto, en un estudio entre estudiantes femeninas de licenciatura en Estados Unidos de América (EUA), ${ }^{35}$ se encontró que las que habían sufrido abuso sexual en la niñez tuvieron dos veces más la posibilidad de sufrir violencia sexual en la vida adulta. Por otra parte, en su estudio Jankowski y colaboradores, ${ }^{35}$ encontraron que cuando existió tanto violencia física como sexual durante la niñez de las jóvenes, fue tres veces más posible su revictimización sexual.

Un estudio realizado entre usuarias de servicios de salud en Inglaterra tuvo resultados muy semejantes a los del presente estudio, ya que registró asociaciones

\section{Cuadro IV}

Efecto de la violencia durante la niñez en la violación* en la vida adulta. ENVIM 2003, México

\begin{tabular}{|c|c|c|c|c|}
\hline Características & $\begin{array}{l}\text { Ausencia } \\
\text { Núm. (\%) }\end{array}$ & $\begin{array}{l}\text { Presencia } \\
\text { Núm. (\%) }\end{array}$ & RM cruda (IC95\%) & RM ajustada $($ IC95\%) \\
\hline \multicolumn{5}{|c|}{ Golpes durante la niñez } \\
\hline Nunca & 14766 (58.7) & $287(32.7)$ & 1.0 & 1.0 \\
\hline Pocas veces & 60 I5। (24.4) & $23 I(26.3)$ & $1.9(1.6-2.3)$ & $1.6(1.3-1.9)$ \\
\hline Varias veces & $2264(9.0)$ & $148(16.9)$ & $3.4(2.7-4.1)$ & $2.2(1.8-2.8)$ \\
\hline Casi siempre & I 984 (7.9) & $2 I I(24.1)$ & $5.5(4.5-6.6)$ & $2.9(2.4-3.6)$ \\
\hline \multicolumn{5}{|c|}{ Abuso sexual ( $<15$ años de edad) } \\
\hline No & $23690(94.1)$ & $446(50.9)$ & 1.0 & 1.0 \\
\hline Sí & I 475 (5.9) & $43 I(49.1)$ & $15.5(13.5-17.9)$ & $11.8(10.2-13.7)$ \\
\hline
\end{tabular}


significativas entre el abuso sexual o físico durante la niñez y la violencia física y sexual, así como la violación, por parte de una persona diferente a la pareja durante la edad adulta. ${ }^{36}$ Otro estudio entre pacientes psiquiátricas femeninas en EUA, encontró una asociación entre mujeres con el antecedente de abuso sexual o violencia física durante la niñez y la experiencia de la violencia sexual durante la edad adulta. ${ }^{37}$ Aunque en un estudio realizado en mujeres reclutadas para trabajar en la marina estadounidense se encontró una fuerte asociación entre el abuso sexual durante la niñez y la violación en la edad adulta, no se registró una asociación entre el abuso físico durante la niñez y la violación. ${ }^{47}$ Un estudio realizado en una muestra representativa en el plano nacional en EUA, encontró, de manera similar al presente estudio realizado en México, que las mujeres que habían sido víctimas de abuso físico o sexual durante la niñez estaban en riesgo de ser víctimas de violencia física y sexual una vez que eran adultas, tanto por parte de sus parejas como de otras personas. ${ }^{48} \mathrm{La}$ existencia de hallazgos semejantes en sociedades tan distintas como México, EUA, Inglaterra y Alemania pareciera indicativo de la magnitud que este problema representa en el ámbito mundial.

El fenómeno de revictimización, o repetición de la violencia en más de una etapa en la vida de las mujeres, pareciera indicar que la violencia durante la niñez tiene efectos duraderos en el desarrollo emocional y social del individuo. Por ejemplo, se ha indicado que cuando las mujeres son víctimas de violencia durante la niñez, se afecta el estado psicológico de las mismas en etapas posteriores de su vida. ${ }^{4,5,6}$ Los efectos psicológicos o sociales podrían ser los detonantes de que las mujeres sean vulnerables a la revictimización. Sin embargo, para confirmar esta hipótesis será necesario realizar otros estudios diferentes al actual (transversal). Por ejemplo, para confirmar efectivamente cuál fue la causa de la violencia en la etapa adulta, probablemente se requieran estudios longitudinales que siguen a los sujetos a lo largo de sus vidas. Por otra parte, los estudios cualitativos de diversos tipos, por ejemplo, con grupos de mujeres que experimentaron la revictimización, así como las que sólo sufrieron violencia en la niñez, las que solamente enfrentaron violencia en la vida adulta y las que no han sido víctimas de violencia interpersonal, serían útiles para entender más sobre los significados de la violencia, así como para generar nuevas hipótesis que luego podrían ser investigadas a través de metodologías cuantitativas.

\section{Limitaciones}

Una de las limitaciones de este estudio es que la población de mujeres entrevistadas es representativa de las usuarias de los servicios públicos de salud; por lo tanto, los resultados sólo se pueden extrapolar a esta población. Es posible que la continuidad de violencia entre estas mujeres que buscaban algún tipo de atención médica fuera más alta que en otras poblaciones, dado que las personas que experimentan violencia suelen tener un peor estado de salud que quienes no sufren este tipo de victimización. Por otra parte, debido a que las mujeres son usuarias de servicios públicos de salud, puede existir un sesgo hacia la población con menores recursos, y esto debe ser considerado al extrapolarse los resultados a las usuarias de servicios de salud privados. Sin embargo, no todas las usuarias de los servicios públicos presentan una situación socioeconómica tan desventajosa, así que la muestra no está del todo sesgada hacia la población más pobre del país.

Por otra parte, en cuanto a la violencia durante la niñez, únicamente se preguntó si la persona fue golpeada durante la niñez, sin especificar con qué u otros detalles sobre el tipo de abuso físico que experimentó. Por lo tanto, se carece de datos para medir la gravedad de la violencia física padecida. Asimismo, el cuestionario recolectó información sobre la violencia que había ocurrido décadas antes durante la niñez, por lo cual pudiera haber sesgo de memoria. Sin embargo, este sesgo tendería a subestimar la prevalencia, además de que es aleatorio. Finalmente, al ser este un estudio transversal, es imposible hacer conclusiones sobre la causalidad de los factores y fenómenos estudiados.

\section{Conclusiones}

La violencia interpersonal durante la niñez crea un cuadro de resultados negativos, tanto físicos como psicológicos y sociales, como se indica en el presente artículo. Es decir, la experiencia de la violencia durante la ninez tiene como efecto posterior la revictimización o que suceda la violencia también en la edad adulta. Esto constituye un elemento más que nos indica que la violencia durante la niñez, obviamente la sexual, pero también la física, es algo que se debe prevenir y que, cuando se detecta el problema, requiere ser atendido y resuelto de la manera más pronta posible. Esto es especialmente importante dado que, como indica este estudio, una mayor frecuencia del abuso físico o sexual durante la niñez implica una asociación más fuerte con la experiencia de la violencia durante la etapa adulta. De manera general, este estudio indica que el maltrato físico y sexual en la niñez tiene un efecto negativo a largo plazo en la vida de las víctimas. Esto implica que cuando los trabajadores de la salud detectan que alguna usuaria ha sufrido abuso durante la niñez, presenta 
un mayor riesgo de sufrir varios tipos de violencia durante la vida adulta. Por lo tanto, existe la oportunidad y la obligación de prevenir la revictimización o, al menos, detectarla y resolverla.

\section{Referencias}

I. Cloitre M, Cohen LR, Edelman RE, Han H. Posttraumatic stress disorder and extent of trauma exposure as correlates of medical problems and perceived health among women with childhood abuse. Women Health 200I;34(3): I-I7.

2. Taylor RR, Jason LA. Chronic fatigue, abuse-related traumatization, and psychiatric disorders in a community-based sample. Soc Sci Med 2002;55:247-256

3. Drossman DA, Talley NJ, Leserman J, Olden KW, Barreiro MA. Sexual and physical abuse and gastrointestinal illness: review and recommendations. Ann Intern Med 1995; 123:782-794.

4. Flannery DJ, Singer MI,Wester K. Violence exposure, psychological trauma, and suicide risk in a community sample of dangerously violent adolescents. J Am Acad Child Adolesc Psychiatry 200 I;40(4):435-442. 5. DuRant RH,Altman D, Wolfson M, Barkin S, Kreiter S, Krowchuk D. Exposure to violence and victimization, depression, substance use, and the use of violence by young adolescents. J Pediatr 2000; I37:707-7I3. 6. Hill J, Pickles A, Burnside E, Byatt M, Rollinson L, Davis R et al. Child sexual abuse, poor parental care and adult depression: evidence for different mechanisms. Br J Psychiatry 200 I; I79:104-109.

7. McHolm AE, MacMillan HL, Jamieson E. The relationship between childhood physical abuse and suicidality among depressed women: results from a community sample. Am J Psychiatry 2003;160:933-938. 8. Brodsky BS, Oquendo M, Ellis SP, Haas GL, Malone KM, Mann JJ.The relationship of childhood abuse to impulsivity and suicidal behavior in adults with major depression. Am J Psychiatry 200 I; | 58: I87|- I877.

9. Cleary SD.Adolescent victimization and associated suicidal and violent behaviors. Adolescence 2000;35(I40):67|-682.

I0. Johnson JG, Cohen P, Gould MS, Kasen S, Brown J, Brook JS. Childhood adversities, interpersonal difficulties, and risk for suicide attempts during late adolescence and early adulthood. Arch Gen Psychiatry 2002;59:741-749.

I I. Nichols HB, Harlow BL. Childhood abuse and risk of smoking onset. J Epidemiol Comunity Health 2004;58:402-406.

I2. Heffernan K, Cloitre M, Tardiff K, Marzuk PM, Portera L, Leon AC. Childhood trauma as a correlate of lifetime opiate use in psychiatric patients. Addict Behav 2000;25(5):797-803.

I3. Wyatt GE, Guthrie D, Notgrass CM. Differential effects of women's child sexual abuse and subsequent sexual revictimization. J Consult Clin Psychol 1992;60(2):I67-I73.

I4. Maman S, Campbell J, Sweat MD, Gielen AC. The intersections of $\mathrm{HIV}$ and violence: directions for future research and interventions. Soc Sci Med 2000;50(4):459-478.

15. Fergusson MD, Horwood JL, Lynskey TL. Childhood sexual abuse, adolescent sexual behaviors and sexual revictimization. Child Abuse Negl 1997;2I(8):789-803.

16. Ramírez Rodríguez JC, Uribe Vázquez G. Mujer y violencia: Un hecho cotidiano. Sal Pub Mex 1993;35(2): I48-I60.

17. Ramos BM, Carlson BE, McNutt LA. Lifetime abuse, mental health, and African American women. J Fam Violence 2004; 19(3): I53-I 64.
18. Gilbert L, El-Bassel N, Schilling R, Friedman E. Childhood abuse as a risk for partner abuse among women in methadone maintenance.Am J Drug Alcohol Abuse 1997;23:58I-595.

19. Irwin HJ.Violent and nonviolent revictimization of women abused in childhood. J Interpers Violence 1999; I4:1095-III0.

20. Kemp A, Rawlings El, Green BL. Post-traumatic stress disorder (PTSD) in battered women: a shelter sample. J Trauma Stress |99|;4:137-|48.

21. Briere J, Runtz M. Post sexual abuse trauma: data and implications for clinical practice. J Interpers Violence 1987;2:367-379.

22. Gorcey M, Santiago JM, McCall-Perez F. Psychological consequences for women sexually abused in childhood. Soc Psychiatry 1986;21:I29-I33. 23. Alexander PC, Lupfer SL. Family characteristics and long-term consequences associated with sexual abuse. Arch Sex Behav 1987; 16:235-245.

24. Gidycz CA, Coble CN, Latham L, Layman JM. Sexual assault experience in adulthood and prior victimization experiences: a prospective analysis. Psychol Women Q 1993; I7:I5I-I68. 25. Dutton MA, Burghardt KJ, Perrin SG, Chrestman KR, Halle PM. Battered women's cognitive schemata. J Trauma Stress 1994;7:237-255. 26. Stevenson MR, Gajarsky WM. Unwanted childhood sexual experiences relate to later revictimisation and male perpetration. J Psychol Human Sex 1992;4:57-70.

27. Jackson JL, Calhoun KS, Amick AE, Madderer HM, Habif VL. Young adult women who report intrafamilial sexual abuse: subsequent adjustment. Arch Sex Behav 1990;19:21 I-221.

28. Koss MP, Dinero TE. Discriminant analysis of risk factors for sexual victimisation among a national sample of college women. J Consult Clin Psychol 1989;57:242-250.

29. Mayall A, Gold SR. Definitional issues and mediating variables in the sexual revictimisation of women sexually abused as children.J Interpers Violence 1995; 10:26-42.

30. Mandoki CA, Burkhart BR. Sexual victimization: is there a vicious cycle:Violence Vict 1989;4:179-190.

3I. Kilpatrick DG, Acierno R, Resnick HS, Saunders BE, Best CL. A 2 year longitudinal analysis of the relationship between violent assault and substance use in women. J Consult Clin Psychol 1997;65:834-847.

32. Beichman JH, Zucker JK, Hood JE, DaCosta GA.A review of the long-term effects of child sexual abuse. Child Abuse Negl 1992;16(1):101-118.

33. Urquiza AJ, Goodlin-Jones BL. Child sexual abuse and adult revictimization with women of color.Violence Vict 1994;9(3):223-232.

34. Krahe B, Scheinberger-Olwig R, Waizenhofer E, Kolpin S. Childhood sexual abuse and revictimization in adolescence. Child Abuse Negl 1999;23(4):383-394.

35. Jankowski MK, Leitenberg H, Henning K, Coffey P. Parental caring as a possible buffer against sexual revictimization in young adult survivors of child sexual abuse. J Trauma Stress 2002;15(3):235-244.

36. Coid J, Petruckevitch A, Feder G, Chung WS, Richardson J, Moorey S. Relation between childhood sexual and physical abuse and risk of revictimisation in women: a cross-sectional survey. Lancet 200I;358:450-454

37. Cloitre M, Tardiff K, Marzuk PM, Leon AC, Portera L. Childhood abuse and subsequent sexual assault among female inpatients. J Trauma Stress 1996;9(3):473-482.

38. Desai S,Arias I, Thompson MP, Basile KC. Childhood victimization and subsequent adult revictimization assessed in a nationally representative sample of women and men.Violence Vict 2002; 17(6):639-653.

39. Alvarado-Zaldívar G, Salvador-Moysén J, Estrada-Martínez S, Torrones-González A. Prevalencia de violencia doméstica en la ciudad de Durango. Salud Publica Mex 1998;40:481-486. 
40. Castro R, Ruiz A. Prevalencia y severidad de la violencia contra mujeres embarazadas, México. Saude Publica 2004;38:62-70. 4I. Rivera-Rivera L, Lazcano-Ponce E, Salmerón-Castro J, Salazar-Martínez E, Castro R, Hernández-Ávila M. Prevalence and determinants of male partner violence against Mexican women:A population-based study. Salud Publica Mex 2004;46: I I3-I 22.

42. Encuesta Nacional sobre Violencia contra las Mujeres 2003. (ENVIM, 2003). Cuernavaca, México: Instituto Nacional de Salud Pública, 2003.

43. Hudson W, Mcintosh S. The assessment of spouse abuse: Two quantifiable dimensions. J Marriage Fam 1981;43:873-885

44. Marshall L. Development of the severity of violence against women scales.J Fam Violence 1992;7:103-121.
45. Valdez-Santiago R, Hijar-Medina M, Salgado N, Rivera-Rivera L,AvilaBurgos L, Olaíz-Fernández G. Escala de violencia e índice de severidad: una propuesta metodológica para la medición de la violencia de pareja en mujeres mexicanas. Salud Publica Mex 2006;48 supl 2:S22I-S23I.

46. Olaiz G, Franco A, Palma O, Echarri C,Valdez R, Herrera C. Diseño metodológico de la Encuesta Nacional sobre Violencia contra las Mujeres en México. Salud Publica Mex 2006;48 supl 2:S328-S335. 47. Merrill LL, Newell CE, Thomsen C], Gold SR, Milner JS, Koss MP et al. Childhood abuse and sexual revictimization in a female Navy recruit sample.J Trauma Stress 1999;12(2):21 I-225

48. Desai S,Arias I,Thompson MP, Basile KC. Childhood victimization and subsequent adult revictimization assessed in a nationally representative sample of women and men.Violence Vict 2002;17(6):639-653. 
Anexo I

Reactivos QUe miden los tipos de VIOLENCIA EN CONTRA DE LA MUJER POR PARTE del esposo, pareja o compañero. ENVIM 2003, México

\begin{tabular}{|c|c|c|c|c|}
\hline Reactivos & \multirow{2}{*}{$\begin{array}{c}\text { Muchas veces } \\
\text { (I) }\end{array}$} & \multirow{2}{*}{$\begin{array}{c}\text { Varias veces } \\
\text { (2) }\end{array}$} & \multirow{2}{*}{$\begin{array}{c}\text { Alguna vez } \\
\text { (3) }\end{array}$} & \multirow{2}{*}{$\begin{array}{c}\text { Nunca } \\
\text { (4) }\end{array}$} \\
\hline a) ¿Le ha rebajado o menospreciado? & & & & \\
\hline b) ¿La menosprecia o humilla frente a otras personas? & & & & \\
\hline c) ¿Le ha insultado? & & & & \\
\hline d) ¿Se ha puesto celoso o ha sospechado de sus amistades? & & & & \\
\hline e) ¿Le ha dicho cosas como que sea usted poco atractiva o fea? & & & & \\
\hline f) ¿Se ha puesto a golpear o patear la pared o algún mueble? & & & & \\
\hline g) ¿Le ha destruido alguna de sus cosas? & & & & \\
\hline h) ¿Le ha amenazado con golpearle? & & & & \\
\hline i) ¿Le ha amenazado con alguna navaja, cuchillo o machete? & & & & \\
\hline j) ¿Le ha amenazado con alguna pistola o rifle? & & & & \\
\hline k) ¿Le ha hecho sentir miedo de él? & & & & \\
\hline I) ¿Le ha amenazado con matarla o matarse él o a los niños? & & & & \\
\hline II) ¿Le ha empujado a propósito? & & & & \\
\hline m) ¿Le ha sacudido, zarandeado o jaloneado? & & & & \\
\hline n) iLe ha torcido el brazo? & & & & \\
\hline ñ) ¿Le ha pegado con la mano o con el puño? & & & & \\
\hline 0) ¿Le ha pateado? & & & & \\
\hline p) ¿Le ha golpeado con algún palo o cinturón o algún objeto domésti & & & & \\
\hline q) ¿Le ha quemado con cigarro o cualquier otra sustancia? & & & & \\
\hline r) ¿Le ha tratado de ahorcar o asfixiar? & & & & \\
\hline s) ¿Le ha agredido con alguna navaja, cuchillo o machete? & & & & \\
\hline t) ¿Le ha disparado con una pistola o rifle? & & & & \\
\hline u) ¿La ha controlado con no darle dinero o quitándoselo? & & & & \\
\hline v) ¿Le ha quitado o ha hecho uso de sus pertenencias en contra de $s$ & & & & \\
\hline w) ¿Le ha exigido tener relaciones sexuales? & & & & \\
\hline $\begin{array}{l}\text { x) ¿Le ha amenazado con irse con otras mujeres si no accede a tener } \\
\text { relaciones sexuales con él? }\end{array}$ & & & & \\
\hline y) ¿Ha usado fuerza física para tener relaciones sexuales con usted? & & & & \\
\hline
\end{tabular}

